\title{
A SIMPLE MODEL TO OPTIMIZE THIRD INTEGER RESONANCE EXTRACTION PARAMETERS
}

\author{
Vladimir Nagaslaev and Leo Michelotti, Fermilab*，Batavia, IL 60510, USA
}

\section{Abstract}

In storage rings with slow resonant extraction one of the main goals of optimization is the extraction inefficiency, or the fraction of beam lost due to hitting the septum plane. A simple analytical model based on a perturbation theoretic approach is built to help determine optimal parameters. It also helps to evaluate the design performance in time and in a range of machine parameters.

\section{INTRODUCTION}

The third-integer resonance is widely used for slowly extracting a good quality beam continuously over an extended time period, the "spill." The range of possible applications is wide, as well as the range of requirements imposed by each project. Due to the high complexity, performance optimization at the design stage is best accomplished with tracking simulations that may include machine optics specific details, beam properties, space charge effects, RF feedback loops and so forth. However, it would be practical to use simple, analytic calculations to evaluate the reach of main performance characteristics and reduce the allowed range of machine parameters before doing rigorous simulations. This possibility is offered by a simple model based on perturbation theory and a few reasonable assumptions. The model was developed in the context of slow extraction from the Fermilab Delivery Ring ${ }^{1}$ for the Mu2e experiment.

\section{RESONANCE MODEL}

Figure 1 illustrates the idealized separatrix for the thirdinteger resonance drawn in a complexified, normalized, horizontal phase space with coordinates,

$$
a=\sqrt{I} e^{i(\pi / 2-\varphi)}=\frac{x+i\left(\alpha x+\beta x^{\prime}\right)}{\sqrt{2 \beta}}
$$

where $\beta$ and $\alpha$ are the usual Courant-Snyder horizontal lattice functions. The Hamiltonian associated with the thirdinteger resonance model, written in angle-action coordinates $(\varphi, I)$, is as follows. $[1,2]$

$$
\begin{aligned}
H & =\Delta \nu \cdot a^{*} a-i g a^{3}+i g^{*} a^{* 3}+\cdots \\
& =\Delta \nu I-\left(g e^{-i 3 \varphi}+g^{*} e^{i 3 \varphi}\right) I^{3 / 2}
\end{aligned}
$$

For Mu2e extraction, the difference between the linear (i.e. small amplitude) horizontal tune and the resonant tune is

\footnotetext{
* Operated by Fermi Research Alliance, LLC under Contract No. DEAC02-07CH11359 with the United States Department of Energy.

${ }^{1}$ Formerly, the antiproton Debuncher Ring.
}

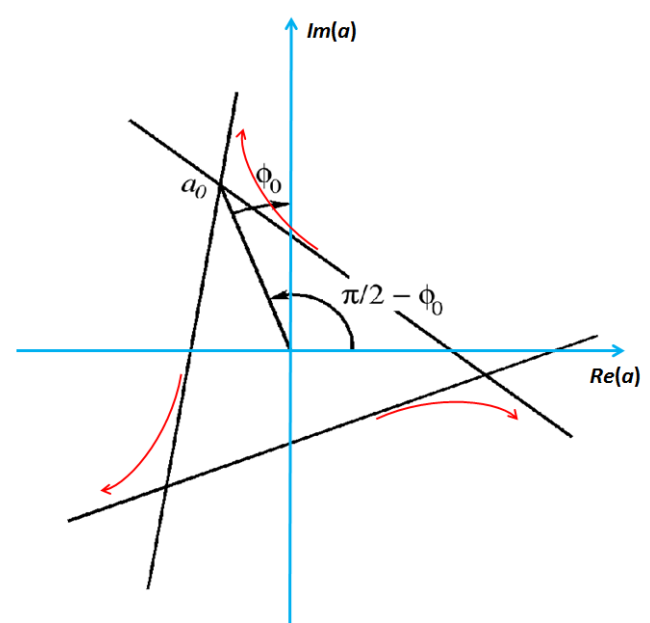

Figure 1: Separatrix geometry.

$\Delta \nu=\nu_{x}-29 / 3$, which is presumed to be small. The "resonance coupling constant," $g$, is a linear functional of the sextupole field strength distribution,

$$
g=\frac{i}{6 \sqrt{2}} \frac{1}{4 \pi} \sum\left(\frac{B^{\prime \prime} l}{B \rho} \beta^{3 / 2}(\theta) e^{-i 3(\psi(\theta)-\Delta \nu \theta)}\right)
$$

where the sum is carried out over the locations of the sextupoles. The phase, $\Psi$, of the complex parameter $g$ determines the orientation of the third-integer separatrix, which is bound by the equilateral triangle with vertices

$$
\begin{aligned}
\left|a_{0}\right| & =\sqrt{I_{0}}=|\Delta \nu / 3 g| \\
0<\Delta \nu & \Rightarrow \varphi_{0}=\Psi / 3 \bmod 2 \pi / 3 \\
\Delta \nu<0 \Rightarrow \varphi_{0} & =\Psi / 3+\pi / 3 \bmod 2 \pi / 3 .
\end{aligned}
$$

as shown in Figure 1. In the beginning of extraction all beam should be included in the starting separatix - this determines initial $\Delta \nu$ and $g$. As $\Delta \nu$ reduces during extraction, the separatrix boundary squeezes leaving less and less stable beam in the machine. Particles outside the separatrix are streaming away along the outcoming rays of the triangle like shown by arrows in Figure 1. The direction of streaming depends on the sign of $\Delta \nu$; the figure illustrates a flow when $\Delta \nu<0$.

\section{STEP SIZE}

The step size is a measure of the speed of a particle streaming away and is defined as the increase in its horizontal projection after three consecutive turns. In order to estimate the step size we assume that (a) the extraction process is adiabatic and (b) extracted particles reside on the 
outgoing branch of the separatrix. The latter approximation is reasonable provided they are not "too far" from it. Accordingly, we write

$$
a \approx a_{0}\left(1+r e^{\operatorname{sgn}(\Delta \nu) i \pi / 6}\right) .
$$

Transformation to the $\mathrm{x}$-coordinate can be written as

$$
\begin{aligned}
x & =\sqrt{2 \beta} \Re\left\{a_{0}\left(1+r e^{\operatorname{sgn}(\Delta \nu) i \pi / 6}\right)\right\}, \\
\Delta x & =\sqrt{2 \beta} \Re\left\{a_{0} e^{\operatorname{sgn}(\Delta \nu) i \pi / 6}\right\} \cdot \Delta r .
\end{aligned}
$$

The step size is determined from $d a / d \theta=-i \partial H / d a^{*}$, which yields

$$
d r / d \theta=|\Delta \nu| \cdot r(\sqrt{3}+r) .
$$

We integrate this over three turns to obtain the result,

$$
\Delta r=\frac{r(r+\sqrt{3})}{[\sqrt{3} /(\exp (6 \pi \sqrt{3}|\Delta \nu|)-1)]-r} .
$$

Notice that the sextupole strength does not appear explicitly in these expressions. Instead, it is present implicitly in the parameter $a_{0}$, which actually has to be matched initially to the injected beam size. Eq.(3) is used to fix the initial ratio $|\Delta \nu / g|$ at injection, but there is some freedom of increasing the sextupole strength while moving the initial machine tune farther away from the resonance.

\section{OPTIMIZATION OF THE EXTRACTION EFFICIENCY}

Eq.(5) and Eq.(1) imply $|\Delta x| \leq \sqrt{2 \beta}\left|a_{0}\right| \Delta r$, with equality when $\left(\pi / 2-\varphi_{0}\right)+\operatorname{sgn}(\Delta \nu) \pi / 6=0$ or $\pi$. (Of necessity, $\Delta r \geq 0$ while $\Delta x$ may be positive or negative.) For $\Delta \nu<0$ and a given $\left|a_{0}\right|$, the ratio $|\Delta x| / \Delta r$ is therefore maximized by orienting the separatrix so that $\varphi_{0}=-2 \pi / 3$ when $\Delta x<0$ on the outgoing branch, thereby making it parallel to the $x$-axis. ${ }^{2}$ We shall assume this orientation, where needed, in what follows. Eq.(4) then simplifies to

$$
x=x_{0} \cdot(1+2 r / \sqrt{3}) \text {, with } x_{0}=-\sqrt{3 \beta / 2}\left|a_{0}\right| .
$$

With this configuration, $x \leq x_{0}<0$, accordant with counter-clockwise rotation of the bunch in the Delivery Ring. To avoid having to adjust to this polarity continually, we shall define $X \equiv-x$ and use it below.

Figure 2 shows $X_{1}(r)$, the horizontal position as function of the dimensionless parameter $r$, and $X_{2}(r)$, the horizontal position on the next step, after the next three turns. That is, $X_{2}(r)=X_{1}(r)+\Delta x(r)$, where $r$ is obtained by inverting Eq.(8) with $x \equiv X_{1}$. The value is then used in Eq.(7) to find $\Delta r$, which is then inserted into Eq.(5), which determines $\Delta x$. The lower horizontal dash line in the plot shows the position of the septum wire/foil plane. The beam

\footnotetext{
${ }^{2}$ Maximizing $|\Delta x|$ decreases the probability of hitting the septum's wire or foil. Maximizing the ratio $|\Delta x| / \Delta r$ may not necessarily be optimal but will be close to it.
}

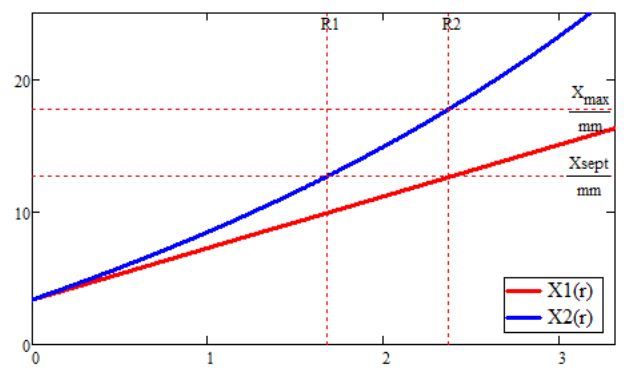

Figure 2: Current x-position $\left(X_{1}\right)$ and x-position after three turns $\left(X_{2}\right)$ as functions of parameter $r$.

particles on their way to extraction span all the space below this plane. Once they pass the vertical marker R1, they would be extracted on the next step as the blue line crosses the septum plane. In the simplified model losses occur for those of them that exactly cross the septum plane thickness, and particles that land on the other side of the wire/foil plane do not contribute to the losses. Although this is a widely used figure of merit for septum losses, the validity of this assumption is limited and varies with the type of septum plane. MARS simulations performed for the Delivery Ring show that this assumption is quite reasonable for thin foil septa. [3]

The larger we can make the gap between red and blue lines (step size), the smaller fraction of particles end up in the septum plane, hence, the better efficiency. However, there are natural limitations on the step size. If it is too large, the particles start to fall out of the machine aperture and have chances to get lost in the ring before reaching the extraction septum. The exact definition of the step size limitation depends on the aperture details of the machine section between the sextupole magnets and the extraction region. Here we require that the blue line should not cross the boundary of machine acceptance, shown as the upper horizontal dash line in Figure 2, which in case of the FNAL Muon Campus Delivery Ring is set to $35 \pi \mathrm{mm}$-mrad. The optimal condition for the septum plane location therefore is obtained by requiring that the blue line cross upper dash line at the same moment when red line crosses the lower dash line (marker R2). This can be optional if the machine acceptance is very large and the step size is limited by other practical factors. However in many cases, including FNAL Delivery ring, machine aperture is a limiting factor. Also, in case of the Delivery ring the condition formulated above is a very close call, as the tightest aperture of the ring is presented in the straight section right upstream of the extraction region.

The fraction of beam loss on the septum's wire (or foil) can be estimated as a ratio

$$
R_{L}=\frac{\left[\int n(x) d x\right]_{w i r e}}{\int_{x_{s e p}}^{x_{\max }} n(x) d x},
$$

where $n$ is the number density of particles on the outgo- 
ing branch of the separatrix. If we assume a steady-state flow, then $n \propto(d r / d \theta)^{-1}$. With this assumption, and using Eq.(6), $R_{L}$ is written,

$$
R_{L}=\frac{d_{w}}{X_{S}^{2}-X_{0}^{2}} \frac{2 X_{0}}{\ln \left(\frac{\left(X_{\max }-X_{0}\right)\left(X_{S}+X_{0}\right)}{\left(X_{\max }+X_{0}\right)\left(X_{S}-X_{0}\right)}\right)},
$$

where the numerator is evaluated only to first order in $d_{w}$, the effective wire plane thickness, assumed to be "small."

\section{PARAMETRIZATION IN TIME}

The plot in Figure 2 is made at a particular moment - at the beginning of the squeeze. As the tune moves to the resonance, the separatrix shrinks (is "squeezed"), which alters the curves $X_{1}(r)$ and $X_{2}(r)$. For the purpose of this discussion, we shall assume that this is accomplished with a dedicated zeroth harmonic quadrupole circuit and that $\Psi$, the phase $g$ appearing in Eq.(2), does not change. This fixes the separatrix's orientation, so we can continue to use Eq.(8) through extraction.

From Eq.(3), the history of a separatrix's vertex is linear in the ramp profile, $\Delta \nu(t)$, where $t$ is time. Thus,

$$
\left|a_{0}(t)\right|=|\Delta \nu(t) / 3 g|=\frac{\Delta \nu(t)}{\Delta \nu(0)}\left|a_{0}(0)\right| .
$$

In writing the final expression, we assume that $|g|$ remains constant - that is, the separatrix is squeezed by changing only the tune control quads, not the sextupoles - and $\Delta \nu$ does not change sign throughout the ramp. Further, with normalization as in Eq.(1), $\left|a_{0}(0)\right|$ is related to the initial, unmatched emittance, $\epsilon_{i}$, through the expression, ${ }^{3}$

$$
\epsilon_{i}=\frac{\pi}{2}\left|a_{0}(0)\right|^{2} .
$$

Combining these with Eq.(8), we can write

$$
x=-(2 r+\sqrt{3}) \cdot \frac{\Delta \nu(t)}{\Delta \nu(0)} \cdot \sqrt{\beta \epsilon_{i} / \pi} .
$$

This is equivalent to Eq.(8) but parametrized so as to make explicit the dependence on time. ${ }^{4}$

The curves $X_{1}(r)$ and $X_{2}(r)$ can now be defined as before but at any arbitrary time during the ramp. As discussed already, the optimal placement of the septum wire is the value of $X_{1}$ when $X_{2}$ is at the machine aperture (or acceptance). However, the septum plane's position can not be changed during the squeeze, so the optimum must be evaluated at the end of the squeeze, when this value reaches its minimum. This could be mitigated by a dynamic orbit bump if this is practical, but here we shall assume otherwise.

\footnotetext{
3 That is, $\epsilon_{i}$ is the emittance associated with a maximal CourantSnyder invariant ellipse, tangent to the separatrix at three points. It is $1 / 4$ the emittance of an ellipse passing through the vertices, and $\pi /(3 \sqrt{3})$ times the emittance of the central stable region. $\epsilon_{i}$ is an estimate of the injected emittance, before filamentation occurs.

${ }^{4}$ We can neglect the small variation in $\beta$ during the ramp, one of the advantages of third-integer over half-integer extraction.
}

Superficially, the end of the squeeze appears to present a numerical problem because expressions such as those appearing in Eqs.(10), (7) or (6) become meaningless if one blindly plugs in $\Delta \nu=0$. The difficulty arises because the scale of $r$ is set by $a_{0}$, which means that $r \rightarrow \infty$ as $a_{0} \rightarrow 0$, so that $x$ remains finite. It can be handled by defining a "renormalized" real variable, $u$, as $a_{0} r \equiv u \exp \left(-i\left(\varphi_{0}-\pi / 2\right)\right)$. Then, in the limit $\Delta \nu \rightarrow 0$, Eq.(7) approaches the finite expression, ${ }^{5}$

$$
\Delta u=\frac{18 \pi|g| u^{2}}{1-18 \pi|g| u} .
$$

Having thus positioned an optimal $X_{S}=X_{\text {septum }}$, Eq.(9) is used to estimate the loss rate throughout the squeeze by setting $X_{\max }=X_{\text {septum }}+\Delta X$ and using Eq.(10) to fix $X_{0}$. This gives us the loss rate as a function of time, via the ramp profile, and the other two paremeters appearing in Eq.(10).

$$
R_{L}=R_{L}\left(\Delta \nu(t), \epsilon_{i}, \beta\right)
$$

This expression can be obtained by plugging all the parameters as functions of time, as determined above, into Eq.(9). It is straightforward but bulky, so we shall not reproduce it here.

Figures 3 and 4 show the application of this model to Fermilab's Delivery Ring. The tune ramp profile, $\Delta \nu(t)$,

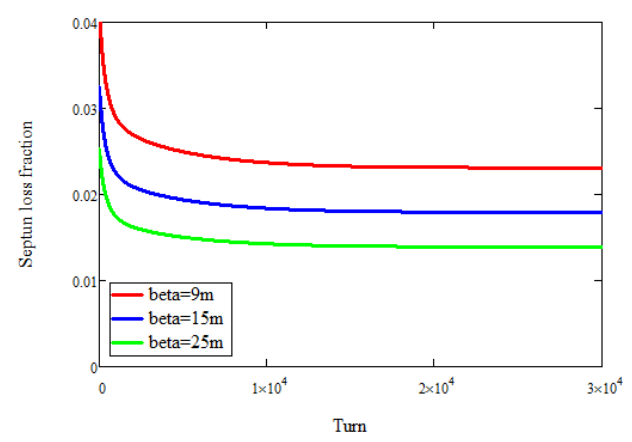

Figure 3: Calculated septum losses as function of time for three values of the beta-function. Initial normalized emittance is $16 \pi \mathrm{mm}-\mathrm{mr}$ and machine acceptance is $35 \pi \mathrm{mm}$ $\mathrm{mr}$.

can be written analytically only for a limited set of simple cases. When space charge, chromaticity and other effects are taken into account, it must be obtained from tracking simulations. In these calculations we used a tune ramp profile devised to produce a close to uniform spill rate in simulations with the program ORBIT. ${ }^{6}$ Figure 3 uses this curve to show losses vs time (parametrized by turn number) for three different values of the beta function. The

\footnotetext{
5 This is most easily seen by first taking the limit of the differential equation, Eq.(6). Details are worked out in Reference [1].

6 More recently, piecewise linear ramp profiles have been designed to minimize deviation from a constant spill rate simulated by the code Synergia. That work will be reported elsewhere.
} 


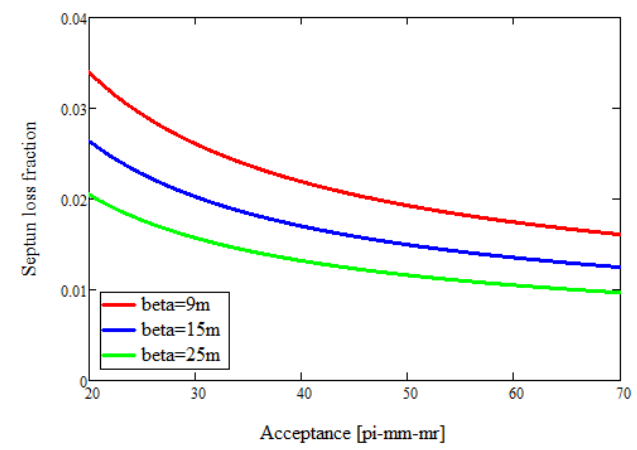

Figure 4: Septum wire losses vs. machine acceptance.

Delivery ring was originally designed as a compact, low beta machine. If its optics remain unchanged during Mu2e operations, the beta function at the entrance to the electrostatic septum would be only $\approx 9$ meters, and the best achievable loss fraction for a $100 \mu \mathrm{m}$ septum wire width would be around $2.5 \% .^{7}$ In order to reduce losses to $2 \%$, it will be necessary to increase $\beta$ above 15 meters.

Figure 4 explores how the machine acceptance improvement could help reduce the septum losses. Losses are calculated at the moment of $30 \%$ through the spill at three values of beta-function. One can see here that doubling the aperture would be necessary in order to achieve the same loss fraction reduction as increasing the beta function by about a factor of three.

\section{SUMMARY}

We described here a semi-analytic approach to calculate the limits of fractional beam losses during third-integer resonance extraction. The parametric calculations are general; we have applied them here to Fermilab's Delivery Ring for slow extraction to the Mu2e experiment.

\section{REFERENCES}

[1] Leo Michelotti and John Johnstone. Preliminaries toward studying resonant extraction from the debuncher. FERMILAB-FN-0842-APC-CD. June, 2009. (Available electronically at URL http://lss.fnal.gov/archive/testfn/0000/fermilab-fn-0842-apc-cd.pdf.)

[2] Leo Michelotti. Intermediate Classical Dynamics with Applications to Beam Physics. John Wiley \& Sons, Inc., New York, 1995.

[3] Vladimir Nagaslaev, et al. FNAL delivery ring electrostatic septum design optimization with MARS simulations. ( To be published)

\footnotetext{
7 The septum width of $100 \mu \mathrm{m}$ is used here as a standard parameter value, but good quality foils are now available from industry with widths of $50 \mu \mathrm{m}$ and less. Using the larger value also allows for some increased inefficiency due to fabrication (placement) error.
} 